\title{
Application of the Method of Molecular Voronoi-Dirichlet Polyhedra for Analysis of Noncovalent Interactions in Aripiprazole Polymorphs
}

\author{
Viktor N. Serezhkin,* Anton V. Savchenkov \\ Samara National Research University, Samara, Russian Federation \\ *E-mail: serezhkin@samsu.ru
}

The following Tables S1-S3 include the following characteristics of interatomic interactions:

- $k$ - the number of pyramids representing interatomic contacts;

- $d_{\min }$ and $d_{\max }(\AA)$ - distances for the shortest and the longest contact respectively;

- $S\left(\AA^{2}\right)$ - the total surface area of all faces corresponding to the given type of contacts;

- $V\left(\AA^{3}\right)$ - the total volume of all pyramids corresponding to the given type of contacts;

- $\Delta_{S}(\%)$ - partial contributions of contacts to the total surface area of the corresponding faces.

The last column $\Sigma$ shows the cumulative values for each molecule. 
Table S1. Characteristics of chemical bonds in APZ polymorphs (VD polyhedra faces with $\mathrm{RF}=1$ )

\begin{tabular}{|c|c|c|c|c|c|c|c|c|c|}
\hline Form & Refcode & & $\mathrm{H} / \mathrm{C}$ & $\mathrm{C} / \mathrm{C}$ & $\mathrm{H} / \mathrm{N}$ & $\mathrm{C} / \mathrm{N}$ & $\mathrm{C} / \mathrm{O}$ & $\mathrm{C} / \mathrm{Cl}$ & $\Sigma$ \\
\hline \multirow[t]{6}{*}{ I } & MELFIT01 & $k$ & 52 & 40 & 2 & 16 & 6 & 4 & 120 \\
\hline & & $d_{\min }$ & 0.93 & 1.37 & 0.86 & 1.34 & 1.23 & 1.73 & 0.86 \\
\hline & & $d_{\max }$ & 0.97 & 1.54 & 0.86 & 1.47 & 1.39 & 1.74 & 1.74 \\
\hline & & $S$ & 221.31 & 209.51 & 14.35 & 68.62 & 31.05 & 29.46 & 574.29 \\
\hline & & V & 35.21 & 49.16 & 2.06 & 16.25 & 6.76 & 8.50 & 117.93 \\
\hline & & $\Delta_{S}$ & 38.54 & 36.48 & 2.50 & 11.95 & 5.41 & 5.13 & 100.00 \\
\hline \multirow[t]{6}{*}{ II } & MELFIT02 & $k$ & 52 & 40 & 2 & 16 & 6 & 4 & 120 \\
\hline & & $d_{\min }$ & 0.93 & 1.33 & 0.86 & 1.36 & 1.22 & 1.73 & 0.86 \\
\hline & & $d_{\max }$ & 0.97 & 1.56 & 0.86 & 1.47 & 1.40 & 1.76 & 1.76 \\
\hline & & $S$ & 222.40 & 212.09 & 14.39 & 68.80 & 33.22 & 28.82 & 579.73 \\
\hline & & V & 35.39 & 49.90 & 2.06 & 16.27 & 7.31 & 8.37 & 119.30 \\
\hline & & $\Delta_{S}$ & 38.36 & 36.58 & 2.48 & 11.87 & 5.73 & 4.97 & 100.00 \\
\hline \multirow[t]{18}{*}{ III } & MELFIT03 & $k$ & 52 & 40 & 2 & 16 & 6 & 4 & 120 \\
\hline & & $d_{\min }$ & 0.93 & 1.34 & 0.86 & 1.35 & 1.22 & 1.72 & 0.86 \\
\hline & & $d_{\max }$ & 0.97 & 1.57 & 0.86 & 1.47 & 1.44 & 1.77 & 1.77 \\
\hline & & $S$ & 222.48 & 221.01 & 12.32 & 67.22 & 31.48 & 30.03 & 584.54 \\
\hline & & $V$ & 35.40 & 51.86 & 1.76 & 15.95 & 6.94 & 8.70 & 120.62 \\
\hline & & $\Delta_{S}$ & 38.06 & 37.81 & 2.11 & 11.50 & 5.38 & 5.14 & 100.00 \\
\hline & III $a$ & $k$ & 52 & 40 & 2 & 16 & 6 & 4 & 120 \\
\hline & & $d_{\min }$ & 0.93 & 1.35 & 0.86 & 1.35 & 1.22 & 1.72 & 0.86 \\
\hline & & $d_{\max }$ & 0.97 & 1.52 & 0.86 & 1.46 & 1.44 & 1.74 & 1.74 \\
\hline & & $S$ & 225.82 & 223.25 & 11.22 & 65.21 & 31.57 & 26.53 & 583.60 \\
\hline & & V & 35.92 & 52.45 & 1.61 & 15.49 & 6.97 & 7.66 & 120.11 \\
\hline & & $\Delta_{S}$ & 38.69 & 38.25 & 1.92 & 11.17 & 5.41 & 4.55 & 100.00 \\
\hline & $\mathrm{III} b$ & $k$ & 52 & 40 & 2 & 16 & 6 & 4 & 120 \\
\hline & & $d_{\min }$ & 0.93 & 1.34 & 0.86 & 1.374 & 1.22 & 1.73 & 0.86 \\
\hline & & $d_{\max }$ & 0.97 & 1.57 & 0.86 & 1.47 & 1.44 & 1.77 & 1.77 \\
\hline & & $S$ & 219.14 & 218.77 & 13.43 & 69.23 & 31.38 & 33.53 & 585.48 \\
\hline & & V & 34.88 & 51.26 & 1.92 & 16.41 & 6.90 & 9.75 & 121.13 \\
\hline & & $\Delta_{S}$ & 37.43 & 37.37 & 2.29 & 11.83 & 5.36 & 5.73 & 100.00 \\
\hline \multirow[t]{18}{*}{ IV } & MELFIT04 & $k$ & 52 & 40 & 2 & 16 & 6 & 4 & 120 \\
\hline & & $d_{\min }$ & 0.93 & 1.35 & 0.86 & 1.35 & 1.23 & 1.72 & 0.86 \\
\hline & & $d_{\max }$ & 0.97 & 1.52 & 0.86 & 1.47 & 1.43 & 1.73 & 1.73 \\
\hline & & $S$ & 221.23 & 220.08 & 12.30 & 68.15 & 33.06 & 29.23 & 584.05 \\
\hline & & V & 35.21 & 51.59 & 1.76 & 16.15 & 7.29 & 8.39 & 120.39 \\
\hline & & $\Delta_{S}$ & 37.88 & 37.68 & 2.11 & 11.67 & 5.66 & 5.00 & 100.00 \\
\hline & $\mathrm{IV} a$ & $k$ & 52 & 40 & 2 & 16 & 6 & 4 & 120 \\
\hline & & $d_{\min }$ & 0.93 & 1.37 & 0.86 & 1.35 & 1.23 & 1.72 & 0.86 \\
\hline & & $d_{\max }$ & 0.97 & 1.52 & 0.86 & 1.47 & 1.43 & 1.73 & 1.73 \\
\hline & & $S$ & 216.59 & 215.72 & 11.56 & 67.59 & 33.02 & 31.52 & 576.00 \\
\hline & & V & 34.49 & 50.67 & 1.66 & 16.04 & 7.27 & 9.06 & 119.19 \\
\hline & & $\Delta_{S}$ & 37.60 & 37.45 & 2.01 & 11.73 & 5.73 & 5.47 & 100.00 \\
\hline & $\mathrm{IV} b$ & $k$ & 52 & 40 & 2 & 16 & 6 & 4 & 120 \\
\hline & & $d_{\min }$ & 0.93 & 1.35 & 0.86 & 1.35 & 1.23 & 1.72 & 0.86 \\
\hline & & $d_{\max }$ & 0.97 & 1.51 & 0.86 & 1.46 & 1.426 & 1.72 & 1.72 \\
\hline & & $S$ & 225.87 & 224.44 & 13.05 & 68.71 & 33.11 & 26.93 & 592.10 \\
\hline & & V & 35.92 & 52.51 & 1.87 & 16.26 & 7.31 & 7.72 & 121.59 \\
\hline & & $\Delta_{S}$ & 38.15 & 37.91 & 2.20 & 11.60 & 5.59 & 4.55 & 100.00 \\
\hline \multirow[t]{6}{*}{ V } & MELFIT05 & $k$ & 52 & 40 & 2 & 16 & 6 & 4 & 120 \\
\hline & & $d_{\min }$ & 0.93 & 1.37 & 0.86 & 1.35 & 1.23 & 1.73 & 0.86 \\
\hline & & $d_{\max }$ & 0.97 & 1.53 & 0.86 & 1.47 & 1.43 & 1.73 & 1.73 \\
\hline & & $S$ & 221.16 & 212.58 & 13.72 & 67.76 & 31.98 & 30.77 & 577.96 \\
\hline & & V & 35.20 & 49.99 & 1.97 & 16.05 & 7.08 & 8.88 & 119.17 \\
\hline & & $\Delta_{S}$ & 38.27 & 36.78 & 2.37 & 11.72 & 5.53 & 5.32 & 100.00 \\
\hline
\end{tabular}


Table S1 (continued)

\begin{tabular}{|c|c|c|c|c|c|c|c|c|c|}
\hline Form & Refcode & & $\mathrm{H} / \mathrm{C}$ & $\mathrm{C} / \mathrm{C}$ & $\mathrm{H} / \mathrm{N}$ & $\mathrm{C} / \mathrm{N}$ & $\mathrm{C} / \mathrm{O}$ & $\mathrm{C} / \mathrm{Cl}$ & $\Sigma$ \\
\hline \multirow[t]{18}{*}{$\mathrm{VI}$} & MELFIT06 & $k$ & 52 & 40 & 2 & 16 & 6 & 4 & 120 \\
\hline & & $d_{\min }$ & 0.93 & 1.37 & 0.87 & 1.34 & 1.23 & 1.72 & 0.87 \\
\hline & & $d_{\max }$ & 0.97 & 1.52 & 0.91 & 1.47 & 1.43 & 1.74 & 1.74 \\
\hline & & $S$ & 225.18 & 222.86 & 12.39 & 66.45 & 31.96 & 31.73 & 590.57 \\
\hline & & $V$ & 35.82 & 52.25 & 1.83 & 15.76 & 7.05 & 9.15 & 121.86 \\
\hline & & $\Delta_{S}$ & 38.13 & 37.74 & 2.10 & 11.25 & 5.41 & 5.37 & 100.00 \\
\hline & $\mathrm{VI} a$ & $k$ & 52 & 40 & 2 & 16 & 6 & 4 & 120 \\
\hline & & $d_{\min }$ & 0.93 & 1.37 & 0.87 & 1.35 & 1.23 & 1.73 & 0.87 \\
\hline & & $d_{\max }$ & 0.97 & 1.52 & 0.87 & 1.47 & 1.43 & 1.73 & 1.73 \\
\hline & & $S$ & 222.97 & 220.59 & 12.31 & 65.70 & 32.14 & 31.35 & 585.06 \\
\hline & & $V$ & 35.48 & 51.68 & 1.78 & 15.59 & 7.10 & 9.04 & 120.66 \\
\hline & & $\Delta_{S}$ & 38.11 & 37.70 & 2.10 & 11.23 & 5.49 & 5.36 & 100.00 \\
\hline & $\mathrm{VI} b$ & $k$ & 52 & 40 & 2 & 16 & 6 & 4 & 120 \\
\hline & & $d_{\min }$ & 0.93 & 1.37 & 0.91 & 1.34 & 1.23 & 1.72 & 0.91 \\
\hline & & $d_{\max }$ & 0.97 & 1.52 & 0.91 & 1.47 & 1.43 & 1.74 & 1.74 \\
\hline & & $S$ & 227.38 & 225.12 & 12.48 & 67.19 & 31.78 & 32.11 & 596.07 \\
\hline & & $V$ & 36.17 & 52.82 & 1.89 & 15.92 & 7.01 & 9.25 & 123.06 \\
\hline & & $\Delta_{S}$ & 38.15 & 37.77 & 2.09 & 11.27 & 5.33 & 5.39 & 100.00 \\
\hline \multirow[t]{6}{*}{ VII } & MELFIT07 & $k$ & 52 & 40 & 2 & 16 & 6 & 4 & 120 \\
\hline & & $d_{\min }$ & 0.95 & 1.38 & 0.86 & 1.35 & 1.23 & 1.74 & 0.86 \\
\hline & & $d_{\max }$ & 0.99 & 1.53 & 0.86 & 1.47 & 1.44 & 1.74 & 1.74 \\
\hline & & $S$ & 226.40 & 207.82 & 13.20 & 68.01 & 31.57 & 28.89 & 575.89 \\
\hline & & $V$ & 36.79 & 49.23 & 1.90 & 16.18 & 6.99 & 8.36 & 119.44 \\
\hline & & $\Delta_{S}$ & 39.31 & 36.09 & 2.29 & 11.81 & 5.48 & 5.02 & 100.00 \\
\hline \multirow[t]{6}{*}{ VIII } & MELFIT18 & $k$ & 52 & 40 & 2 & 16 & 6 & 4 & 120 \\
\hline & & $d_{\min }$ & 0.95 & 1.37 & 0.88 & 1.35 & 1.23 & 1.74 & 0.88 \\
\hline & & $d_{\max }$ & 0.99 & 1.53 & 0.88 & 1.47 & 1.44 & 1.74 & 1.74 \\
\hline & & $S$ & 219.63 & 204.94 & 13.74 & 68.32 & 32.43 & 28.21 & 567.27 \\
\hline & & V & 35.71 & 48.30 & 2.02 & 16.23 & 7.20 & 8.19 & 117.65 \\
\hline & & $\Delta_{S}$ & 38.72 & 36.13 & 2.42 & 12.04 & 5.72 & 4.97 & 100.00 \\
\hline \multirow[t]{6}{*}{ IX } & MELFIT19 & $k$ & 52 & 40 & 2 & 16 & 6 & 4 & 120 \\
\hline & & $d_{\min }$ & 0.93 & 1.38 & 0.86 & 1.34 & 1.23 & 1.73 & 0.86 \\
\hline & & $d_{\max }$ & 1.00 & 1.56 & 0.86 & 1.47 & 1.43 & 1.73 & 1.73 \\
\hline & & $S$ & 220.55 & 210.76 & 13.28 & 66.64 & 31.68 & 28.06 & 570.98 \\
\hline & & $V$ & 35.33 & 49.76 & 1.91 & 15.83 & 6.99 & 8.09 & 117.91 \\
\hline & & $\Delta_{S}$ & 38.63 & 36.91 & 2.32 & 11.67 & 5.55 & 4.92 & 100.00 \\
\hline
\end{tabular}


Table S2. Characteristics of intramolecular noncovalent interactions in APZ polymorphs (VD polyhedra faces with RF $>1$ )

\begin{tabular}{|c|c|c|c|c|c|c|c|c|c|c|c|c|c|c|c|c|c|c|}
\hline Form & Refcode & & $\mathrm{H} / \mathrm{H}$ & $\mathrm{H} / \mathrm{C}$ & $\mathrm{C} / \mathrm{C}$ & $\mathrm{H} / \mathrm{N}$ & $\mathrm{C} / \mathrm{N}$ & $\mathrm{N} / \mathrm{N}$ & $\mathrm{H} / \mathrm{O}$ & $\mathrm{C} / \mathrm{O}$ & $\mathrm{N} / \mathrm{O}$ & $\mathrm{O} / \mathrm{O}$ & $\mathrm{H} / \mathrm{Cl}$ & $\mathrm{C} / \mathrm{Cl}$ & $\mathrm{N} / \mathrm{Cl}$ & $\mathrm{O} / \mathrm{Cl}$ & $\mathrm{Cl} / \mathrm{Cl}$ & $\Sigma$ \\
\hline \multirow[t]{6}{*}{ I } & MELFIT01 & $k$ & 106 & 136 & 40 & 42 & 18 & & 18 & 4 & & & 6 & 4 & 2 & & 2 & 378 \\
\hline & & $d_{\min }$ & 1.56 & 1.90 & 2.28 & 2.00 & 2.40 & & 1.85 & 2.40 & & & 2.65 & 2.68 & 2.98 & & 3.12 & 1.56 \\
\hline & & $d_{\text {max }}$ & 3.76 & 3.46 & 2.81 & 3.21 & 2.79 & & 2.84 & 1.41 & & & 3.66 & 2.72 & 2.98 & & 3.12 & 3.76 \\
\hline & & $S$ & 254.07 & 71.30 & 3.79 & 36.67 & 2.62 & & 36.97 & 0.24 & & & 17.26 & 0.57 & 2.70 & & 5.70 & 431.90 \\
\hline & & $V$ & 94.09 & 27.19 & 1.54 & 14.39 & 1.08 & & 15.03 & 0.10 & & & 8.22 & 0.26 & 1.34 & & 2.97 & 166.20 \\
\hline & & $\Delta_{S}$ & 58.83 & 16.51 & 0.88 & 8.49 & 0.61 & & 8.56 & 0.06 & & & 4.00 & 0.13 & 0.63 & & 1.32 & 100.00 \\
\hline \multirow[t]{6}{*}{ II } & MELFIT02 & $k$ & 102 & 128 & 44 & 40 & 18 & & 18 & 4 & & & 6 & 6 & 2 & & 2 & 370 \\
\hline & & $d_{\min }$ & 1.57 & 1.91 & 2.35 & 1.97 & 2.41 & & 1.94 & 2.41 & & & 2.64 & 2.67 & 3.00 & & 3.13 & 1.57 \\
\hline & & $d_{\max }$ & 3.44 & 3.43 & 2.83 & 3.07 & 2.78 & & 2.85 & 2.422 & & & 3.77 & 2.70 & 3.00 & & 3.13 & 3.77 \\
\hline & & $S$ & 272.24 & 67.61 & 4.65 & 32.03 & 2.52 & & 32.20 & 0.20 & & & 13.05 & 0.45 & 2.37 & & 6.30 & 433.62 \\
\hline & & $V$ & 99.29 & 25.44 & 1.88 & 12.29 & 1.05 & & 12.76 & 0.08 & & & 5.93 & 0.20 & 1.18 & & 3.28 & 163.38 \\
\hline & & $\Delta_{S}$ & 62.78 & 15.59 & 1.07 & 7.39 & 0.58 & & 7.43 & 0.05 & & & 3.01 & 0.10 & 0.55 & & 1.45 & 100.00 \\
\hline \multirow[t]{18}{*}{ III } & MELFIT03 & $k$ & 104 & 140 & 42 & 40 & 18 & & 17 & 3 & & & 6 & 3 & 2 & & 2 & 377 \\
\hline & & $d_{\text {min }}$ & 1.56 & 1.90 & 2.33 & 1.98 & 2.39 & & 1.99 & 2.32 & & & 2.65 & 2.71 & 2.99 & & 3.11 & 1.56 \\
\hline & & $d_{\text {max }}$ & 3.83 & 3.40 & 2.83 & 3.15 & 2.82 & & 2.87 & 2.39 & & & 3.81 & 2.72 & 3.01 & & 3.12 & 3.83 \\
\hline & & $S$ & 260.59 & 71.81 & 3.82 & 37.89 & 3.10 & & 27.14 & 0.24 & & & 14.03 & 0.14 & 2.53 & & 5.72 & 427.02 \\
\hline & & $V$ & 95.10 & 27.22 & 1.56 & 14.56 & 1.28 & & 10.77 & 0.09 & & & 6.47 & 0.07 & 1.27 & & 2.97 & 161.34 \\
\hline & & $\Delta_{S}$ & 61.03 & 16.82 & 0.89 & 8.87 & 0.73 & & 6.36 & 0.06 & & & 3.28 & 0.03 & 0.59 & & 1.34 & 100.00 \\
\hline & III $a$ & $k$ & 104 & 142 & 40 & 40 & 18 & & 18 & 4 & & & 6 & 2 & 2 & & 2 & 378 \\
\hline & & $d_{\min }$ & 1.56 & 1.90 & 2.36 & 1.99 & 2.39 & & 2.00 & 2.35 & & & 2.65 & 2.72 & 3.01 & & 3.12 & 1.56 \\
\hline & & $d_{\max }$ & 3.38 & 3.40 & 2.83 & 3.11 & 2.77 & & 2.81 & 2.39 & & & 3.74 & 2.72 & 3.01 & & 3.12 & 3.74 \\
\hline & & $S$ & 261.79 & 71.66 & 4.07 & 35.05 & 3.11 & & 27.49 & 0.26 & & & 15.27 & 0.10 & 2.25 & & 5.24 & 426.29 \\
\hline & & V & 95.59 & 27.23 & 1.65 & 13.45 & 1.28 & & 10.88 & 0.10 & & & 7.11 & 0.05 & 1.13 & & 2.72 & 161.18 \\
\hline & & $\Delta_{S}$ & 61.41 & 16.81 & 0.96 & 8.22 & 0.73 & & 6.45 & 0.06 & & & 3.58 & 0.02 & 0.53 & & 1.23 & 100.00 \\
\hline & IIII $b$ & $k$ & 104 & 138 & 44 & 40 & 18 & & 16 & 2 & & & 6 & 4 & 2 & & 2 & 376 \\
\hline & & $d_{\min }$ & 1.57 & 1.93 & 2.33 & 1.98 & 2.41 & & 1.99 & 2.32 & & & 2.70 & 2.71 & 2.99 & & 3.11 & 1.57 \\
\hline & & $d_{\max }$ & 3.83 & 3.34 & 2.80 & 3.15 & 2.82 & & 2.87 & 2.32 & & & 3.81 & 2.71 & 2.99 & & 3.11 & 3.83 \\
\hline & & $S$ & 259.39 & 71.96 & 3.56 & 40.74 & 3.10 & & 26.80 & 0.22 & & & 12.78 & 0.19 & 2.82 & & 6.21 & 427.75 \\
\hline & & $V$ & 94.60 & 27.21 & 1.46 & 15.66 & 1.27 & & 10.66 & 0.08 & & & 5.84 & 0.09 & 1.41 & & 3.22 & 161.50 \\
\hline & & $\Delta_{S}$ & 60.64 & 16.82 & 0.83 & 9.52 & 0.72 & & 6.27 & 0.05 & & & 2.99 & 0.04 & 0.66 & & 1.45 & 100.00 \\
\hline
\end{tabular}


Table S2 (continued)

\begin{tabular}{|c|c|c|c|c|c|c|c|c|c|c|c|c|c|c|c|c|c|c|}
\hline Form & Refcode & & $\mathrm{H} / \mathrm{H}$ & $\mathrm{H} / \mathrm{C}$ & $\mathrm{C} / \mathrm{C}$ & $\mathrm{H} / \mathrm{N}$ & $\mathrm{C} / \mathrm{N}$ & $\mathrm{N} / \mathrm{N}$ & $\mathrm{H} / \mathrm{O}$ & $\mathrm{C} / \mathrm{O}$ & $\mathrm{N} / \mathrm{O}$ & $\mathrm{O} / \mathrm{O}$ & $\mathrm{H} / \mathrm{Cl}$ & $\mathrm{C} / \mathrm{Cl}$ & $\mathrm{N} / \mathrm{Cl}$ & $\mathrm{O} / \mathrm{Cl}$ & $\mathrm{Cl} / \mathrm{Cl}$ & $\Sigma$ \\
\hline \multirow[t]{18}{*}{ IV } & MELFIT04 & $k$ & 104 & 143 & 42 & 38 & 18 & & 18 & 4 & & & 6 & 4 & 2 & & 2 & 381 \\
\hline & & $d_{\min }$ & 1.56 & 1.91 & 2.33 & 1.99 & 2.41 & & 1.99 & 2.33 & & & 2.60 & 2.69 & 2.97 & & 3.12 & 1.56 \\
\hline & & $d_{\max }$ & 3.53 & 3.50 & 2.83 & 3.18 & 2.81 & & 2.80 & 2.40 & & & 3.83 & 2.72 & 3.00 & & 3.14 & 3.83 \\
\hline & & $S$ & 250.72 & 65.60 & 4.12 & 31.76 & 2.58 & & 31.02 & 0.28 & & & 16.98 & 0.23 & 2.36 & & 5.96 & 411.62 \\
\hline & & $V$ & 92.02 & 24.42 & 1.68 & 12.10 & 1.07 & & 12.28 & 0.11 & & & 7.90 & 0.10 & 1.17 & & 3.11 & 155.96 \\
\hline & & $\Delta_{S}$ & 60.91 & 15.94 & 1.00 & 7.71 & 0.63 & & 7.54 & 0.07 & & & 4.13 & 0.06 & 0.57 & & 1.45 & 100.00 \\
\hline & $\mathrm{IV} a$ & $k$ & 104 & 142 & 38 & 36 & 18 & & 18 & 4 & & & 6 & 4 & 2 & & 2 & 374 \\
\hline & & $d_{\min }$ & 1.56 & 1.91 & 2.36 & 1.99 & 2.42 & & 1.99 & 2.33 & & & 2.60 & 2.70 & 3.00 & & 3.12 & 1.56 \\
\hline & & $d_{\max }$ & 3.46 & 3.43 & 2.83 & 3.18 & 2.81 & & 2.78 & 2.40 & & & 3.83 & 2.72 & 3.00 & & 3.12 & 3.83 \\
\hline & & $S$ & 232.44 & 65.13 & 3.98 & 28.40 & 2.63 & & 31.97 & 0.29 & & & 15.64 & 0.21 & 1.59 & & 6.14 & 388.42 \\
\hline & & $V$ & 85.29 & 24.30 & 1.62 & 10.78 & 1.09 & & 12.70 & 0.11 & & & 6.99 & 0.09 & 0.80 & & 3.19 & 146.96 \\
\hline & & $\Delta_{S}$ & 59.84 & 16.77 & 1.03 & 7.31 & 0.68 & & 8.23 & 0.07 & & & 4.03 & 0.05 & 0.41 & & 1.58 & 100.00 \\
\hline & $\mathrm{IV} b$ & $k$ & 104 & 144 & 46 & 40 & 18 & & 18 & 4 & & & 6 & 4 & 2 & & 2 & 388 \\
\hline & & $d_{\text {min }}$ & 1.57 & 1.91 & 2.33 & 1.99 & 2.41 & & 1.99 & 2.34 & & & 2.62 & 2.69 & 2.97 & & 3.14 & 1.57 \\
\hline & & $d_{\max }$ & 3.53 & 3.50 & 2.82 & 3.10 & 2.79 & & 2.80 & 2.40 & & & 3.66 & 2.71 & 2.97 & & 3.14 & 3.66 \\
\hline & & $S$ & 269.00 & 66.08 & 4.26 & 35.12 & 2.54 & & 30.08 & 0.27 & & & 18.32 & 0.25 & 3.13 & & 5.78 & 434.82 \\
\hline & & $V$ & 98.75 & 24.54 & 1.74 & 13.42 & 1.06 & & 11.86 & 0.11 & & & 8.81 & 0.11 & 1.55 & & 3.02 & 164.96 \\
\hline & & $\Delta_{S}$ & 61.86 & 15.20 & 0.98 & 8.08 & 0.58 & & 6.92 & 0.06 & & & 4.21 & 0.06 & 0.72 & & 1.33 & 100.00 \\
\hline \multirow[t]{6}{*}{$\mathrm{V}$} & MELFIT05 & $k$ & 100 & 140 & 44 & 40 & 18 & & 16 & 4 & & & 6 & 4 & 2 & & 2 & 376 \\
\hline & & $d_{\min }$ & 1.57 & 1.91 & 2.37 & 1.99 & 2.41 & & 1.98 & 2.38 & & & 2.60 & 2.69 & 2.99 & & 3.12 & 1.57 \\
\hline & & $d_{\max }$ & 3.53 & 3.49 & 2.81 & 3.06 & 2.78 & & 2.84 & 2.40 & & & 3.75 & 2.72 & 2.99 & & 3.12 & 3.75 \\
\hline & & $S$ & 256.80 & 68.44 & 3.56 & 34.35 & 2.61 & & 28.57 & 0.26 & & & 15.44 & 0.63 & 2.24 & & 6.61 & 419.50 \\
\hline & & $V$ & 93.56 & 25.89 & 1.45 & 13.39 & 1.08 & & 11.41 & 0.10 & & & 7.29 & 0.28 & 1.12 & & 3.44 & 159.01 \\
\hline & & $\Delta_{S}$ & 61.22 & 16.31 & 0.85 & 8.19 & 0.62 & & 6.81 & 0.06 & & & 3.68 & 0.15 & 0.53 & & 1.58 & 100.00 \\
\hline
\end{tabular}


Table S2 (continued)

\begin{tabular}{|c|c|c|c|c|c|c|c|c|c|c|c|c|c|c|c|c|c|c|}
\hline Form & Refcode & & $\mathrm{H} / \mathrm{H}$ & $\mathrm{H} / \mathrm{C}$ & $\mathrm{C} / \mathrm{C}$ & $\mathrm{H} / \mathrm{N}$ & $\mathrm{C} / \mathrm{N}$ & $\mathrm{N} / \mathrm{N}$ & $\mathrm{H} / \mathrm{O}$ & $\mathrm{C} / \mathrm{O}$ & $\mathrm{N} / \mathrm{O}$ & $\mathrm{O} / \mathrm{O}$ & $\mathrm{H} / \mathrm{Cl}$ & $\mathrm{C} / \mathrm{Cl}$ & $\mathrm{N} / \mathrm{Cl}$ & $\mathrm{O} / \mathrm{Cl}$ & $\mathrm{Cl} / \mathrm{Cl}$ & $\Sigma$ \\
\hline \multirow[t]{18}{*}{ VI } & MELFIT06 & $k$ & 104 & 140 & 43 & 37 & 18 & & 18 & 4 & & & 6 & 4 & 2 & & 2 & 378 \\
\hline & & $d_{\min }$ & 1.56 & 1.92 & 2.36 & 1.99 & 2.40 & & 1.99 & 2.33 & & & 2.50 & 2.69 & 2.99 & & 3.09 & 1.56 \\
\hline & & $d_{\max }$ & 3.65 & 3.62 & 2.82 & 3.15 & 2.78 & & 2.83 & 2.40 & & & 3.65 & 2.71 & 3.03 & & 3.10 & 3.65 \\
\hline & & $S$ & 248.00 & 62.19 & 4.07 & 31.10 & 2.58 & & 29.35 & 0.28 & & & 19.34 & 0.39 & 1.93 & & 7.03 & 406.25 \\
\hline & & $V$ & 90.88 & 23.13 & 1.66 & 11.84 & 1.07 & & 11.64 & 0.11 & & & 8.79 & 0.17 & 0.97 & & 3.62 & 153.88 \\
\hline & & $\Delta_{S}$ & 61.05 & 15.31 & 1.00 & 7.66 & 0.64 & & 7.22 & 0.07 & & & 4.76 & 0.10 & 0.48 & & 1.73 & 100.00 \\
\hline & $\mathrm{VI} a$ & $k$ & 104 & 142 & 42 & 36 & 18 & & 18 & 4 & & & 6 & 4 & 2 & & 2 & 378 \\
\hline & & $d_{\min }$ & 1.56 & 1.92 & 2.36 & 2.00 & 2.40 & & 1.99 & 2.33 & & & 2.50 & 2.69 & 3.03 & & 3.10 & 1.56 \\
\hline & & $d_{\max }$ & 3.65 & 3.62 & 2.80 & 3.15 & 2.78 & & 2.75 & 2.38 & & & 3.65 & 2.71 & 3.03 & & 3.10 & 3.65 \\
\hline & & $S$ & 244.91 & 61.18 & 4.08 & 30.52 & 2.46 & & 29.31 & 0.28 & & & 18.34 & 0.59 & 1.11 & & 6.74 & 399.51 \\
\hline & & $V$ & 89.67 & 22.58 & 1.66 & 11.48 & 1.02 & & 11.59 & 0.11 & & & 8.21 & 0.27 & 0.56 & & 3.48 & 150.62 \\
\hline & & $\Delta_{S}$ & 61.30 & 15.31 & 1.02 & 7.64 & 0.62 & & 7.34 & 0.07 & & & 4.59 & 0.15 & 0.28 & & 1.69 & 100.00 \\
\hline & $\mathrm{VI} b$ & $k$ & 104 & 138 & 44 & 38 & 18 & & 18 & 4 & & & 6 & 4 & 2 & & 2 & 378 \\
\hline & & $d_{\min }$ & 1.56 & 1.94 & 2.36 & 1.99 & 2.41 & & 1.99 & 2.35 & & & 2.53 & 2.69 & 2.99 & & 3.09 & 1.56 \\
\hline & & $d_{\text {max }}$ & 3.58 & 3.59 & 2.82 & 3.01 & 2.77 & & 2.83 & 2.40 & & & 3.55 & 2.71 & 2.99 & & 3.09 & 3.59 \\
\hline & & $S$ & 251.09 & 63.19 & 4.07 & 31.69 & 2.70 & & 29.39 & 0.27 & & & 20.35 & 0.18 & 2.76 & & 7.31 & 412.99 \\
\hline & & $V$ & 92.09 & 23.68 & 1.65 & 12.20 & 1.12 & & 11.70 & 0.11 & & & 9.37 & 0.08 & 1.38 & & 3.77 & 157.13 \\
\hline & & $\Delta_{S}$ & 60.80 & 15.30 & 0.98 & 7.67 & 0.65 & & 7.12 & 0.07 & & & 4.93 & 0.04 & 0.67 & & 1.77 & 100.00 \\
\hline \multirow[t]{6}{*}{ VII } & MELFIT07 & $k$ & 104 & 144 & 42 & 42 & 18 & & 18 & 4 & & & 6 & 4 & 2 & & 2 & 386 \\
\hline & & $d_{\min }$ & 1.60 & 1.92 & 2.38 & 2.02 & 2.42 & & 2.01 & 2.39 & & & 2.64 & 2.71 & 2.99 & & 3.13 & 1.60 \\
\hline & & $d_{\text {max }}$ & 3.68 & 3.52 & 2.83 & 3.18 & 2.82 & & 2.87 & 2.41 & & & 3.61 & 2.72 & 2.99 & & 3.13 & 3.68 \\
\hline & & $S$ & 236.84 & 64.48 & 3.60 & 38.71 & 2.98 & & 26.81 & 0.29 & & & 16.16 & 0.12 & 2.49 & & 6.21 & 398.69 \\
\hline & & $V$ & 88.92 & 24.42 & 1.48 & 15.56 & 1.24 & & 10.77 & 0.12 & & & 7.86 & 0.06 & 1.24 & & 3.23 & 154.88 \\
\hline & & $\Delta_{S}$ & 59.41 & 16.17 & 0.90 & 9.71 & 0.75 & & 6.72 & 0.07 & & & 4.05 & 0.03 & 0.63 & & 1.56 & 100.00 \\
\hline \multirow[t]{6}{*}{ VIII } & MELFIT18 & $k$ & 106 & 138 & 44 & 40 & 18 & & 18 & 4 & & & 6 & 2 & 2 & & 2 & 380 \\
\hline & & $d_{\min }$ & 1.60 & 1.93 & 2.36 & 2.02 & 2.42 & & 2.00 & 2.40 & & & 2.66 & 2.70 & 2.98 & & 3.11 & 1.60 \\
\hline & & $d_{\max }$ & 3.82 & 3.45 & 2.83 & 3.20 & 2.81 & & 2.84 & 2.41 & & & 3.63 & 2.70 & 2.98 & & 3.11 & 3.82 \\
\hline & & $S$ & 232.56 & 65.21 & 4.07 & 32.29 & 2.82 & & 32.53 & 0.39 & & & 12.06 & 0.20 & 1.78 & & 5.85 & 389.75 \\
\hline & & $V$ & 86.65 & 24.69 & 1.65 & 12.53 & 1.17 & & 13.39 & 0.15 & & & 5.62 & 0.09 & 0.88 & & 3.03 & 149.85 \\
\hline & & $\Delta_{S}$ & 59.67 & 16.73 & 1.04 & 8.28 & 0.72 & & 8.35 & 0.10 & & & 3.10 & 0.05 & 0.46 & & 1.50 & 100.00 \\
\hline \multirow[t]{6}{*}{ IX } & MELFIT19 & $k$ & 104 & 146 & 46 & 38 & 18 & & 16 & 4 & & & 6 & 4 & 2 & & 2 & 386 \\
\hline & & $d_{\text {min }}$ & 1.55 & 1.90 & 2.37 & 1.98 & 2.40 & & 1.98 & 2.39 & & & 2.73 & 2.70 & 2.96 & & 3.15 & 1.55 \\
\hline & & $d_{\text {max }}$ & 3.49 & 3.26 & 2.83 & 3.28 & 2.86 & & 2.82 & 2.40 & & & 3.86 & 2.73 & 2.96 & & 3.15 & 3.86 \\
\hline & & $S$ & 231.65 & 61.01 & 4.31 & 31.73 & 2.73 & & 29.10 & 0.31 & & & 13.89 & 0.20 & 3.18 & & 5.13 & 383.23 \\
\hline & & $V$ & 84.85 & 22.98 & 1.75 & 12.18 & 1.12 & & 11.66 & 0.12 & & & 6.43 & 0.09 & 1.57 & & 2.69 & 145.44 \\
\hline & & $\Delta_{S}$ & 60.45 & 15.92 & 1.12 & 8.28 & 0.71 & & 7.59 & 0.08 & & & 3.62 & 0.05 & 0.83 & & 1.34 & 100.00 \\
\hline
\end{tabular}


Table S3. Characteristics of intermolecular noncovalent interactions in APZ polymorphs (VD polyhedra faces with RF $=0$ )

\begin{tabular}{|c|c|c|c|c|c|c|c|c|c|c|c|c|c|c|c|c|c|c|}
\hline Form & Refcode & & $\mathrm{H} / \mathrm{H}$ & $\mathrm{H} / \mathrm{C}$ & $\mathrm{C} / \mathrm{C}$ & $\mathrm{H} / \mathrm{N}$ & $\mathrm{C} / \mathrm{N}$ & $\mathrm{N} / \mathrm{N}$ & $\mathrm{H} / \mathrm{O}$ & $\mathrm{C} / \mathrm{O}$ & $\mathrm{N} / \mathrm{O}$ & $\mathrm{O} / \mathrm{O}$ & $\mathrm{H} / \mathrm{Cl}$ & $\mathrm{C} / \mathrm{Cl}$ & $\mathrm{N} / \mathrm{Cl}$ & $\mathrm{O} / \mathrm{Cl}$ & $\mathrm{Cl} / \mathrm{Cl}$ & $\Sigma$ \\
\hline \multirow[t]{6}{*}{ I } & MELFIT01 & $k$ & 156 & 130 & & 14 & & & 18 & 8 & & & 52 & 4 & 2 & 2 & & 386 \\
\hline & & $d_{\min }$ & 2.64 & 2.95 & & 3.19 & & & 2.05 & 4.24 & & & 2.91 & 3.63 & 3.48 & 3.87 & & 2.05 \\
\hline & & $d_{\text {max }}$ & 4.61 & 4.67 & & 3.82 & & & 3.89 & 4.46 & & & 4.64 & 3.67 & 3.48 & 3.87 & & 4.67 \\
\hline & & $S$ & 259.51 & 106.19 & & 11.60 & & & 53.80 & 0.53 & & & 96.64 & 4.44 & 3.31 & 1.60 & & 537.61 \\
\hline & & $V$ & 135.22 & 58.90 & & 6.63 & & & 24.60 & 0.39 & & & 53.55 & 2.71 & 1.92 & 1.03 & & 284.95 \\
\hline & & $\Delta_{S}$ & 48.27 & 19.75 & & 2.16 & & & 10.01 & 0.10 & & & 17.98 & 0.83 & 0.61 & 0.30 & & 100.00 \\
\hline \multirow[t]{6}{*}{ II } & MELFIT02 & $k$ & 154 & 118 & 12 & 12 & 10 & & 24 & 8 & & & 44 & 2 & 2 & 6 & & 392 \\
\hline & & $d_{\min }$ & 2.56 & 3.00 & 3.34 & 2.93 & 3.73 & & 2.01 & 3.73 & & & 2.99 & 3.48 & 3.75 & 3.58 & & 2.01 \\
\hline & & $d_{\max }$ & 4.58 & 4.58 & 4.10 & 3.77 & 4.12 & & 4.33 & 4.04 & & & 4.73 & 3.48 & 3.75 & 4.37 & & 4.73 \\
\hline & & $S$ & 271.85 & 88.55 & 6.07 & 12.87 & 3.88 & & 50.42 & 3.51 & & & 95.45 & 3.12 & 1.69 & 6.46 & & 543.84 \\
\hline & & $V$ & 146.78 & 50.62 & 3.65 & 6.69 & 2.44 & & 22.89 & 2.20 & & & 52.44 & 1.81 & 1.06 & 3.95 & & 294.52 \\
\hline & & $\Delta_{S}$ & 49.99 & 16.28 & 1.12 & 2.37 & 0.71 & & 9.27 & 0.64 & & & 17.55 & 0.57 & 0.31 & 1.19 & & 100.00 \\
\hline \multirow[t]{18}{*}{ III } & MELFIT03 & $k$ & 169 & 122 & 6 & 13 & 2 & & 30 & & & & 44 & 7 & 2 & & 2 & 397 \\
\hline & & $d_{\text {min }}$ & 2.29 & 2.87 & 3.47 & 2.97 & 3.29 & & 2.02 & & & & 2.89 & 3.50 & 3.32 & & 4.40 & 2.02 \\
\hline & & $d_{\text {max }}$ & 5.70 & 5.58 & 3.55 & 4.53 & 3.55 & & 4.53 & & & & 4.66 & 3.91 & 3.71 & & 4.78 & 5.70 \\
\hline & & $S$ & 254.26 & 99.89 & 3.08 & 11.19 & 1.29 & & 61.42 & & & & 99.52 & 4.89 & 4.94 & & 2.88 & 543.35 \\
\hline & & $V$ & 134.50 & 56.35 & 1.93 & 6.02 & 0.72 & & 28.75 & & & & 55.66 & 2.99 & 2.86 & & 2.14 & 291.92 \\
\hline & & $\Delta_{S}$ & 46.79 & 18.38 & 0.57 & 2.06 & 0.24 & & 11.30 & & & & 18.32 & 0.90 & 0.91 & & 0.53 & 100.00 \\
\hline & III $a$ & $k$ & 179 & 124 & 6 & 14 & 2 & & 36 & & & & 40 & 7 & 2 & & & 410 \\
\hline & & $d_{\min }$ & 2.29 & 2.87 & 3.47 & 2.97 & 3.29 & & 2.02 & & & & 2.89 & 3.50 & 3.32 & & & 2.02 \\
\hline & & $d_{\max }$ & 5.70 & 5.58 & 4.55 & 4.01 & 3.55 & & 4.18 & & & & 4.66 & 3.91 & 3.71 & & & 5.70 \\
\hline & & $S$ & 248.52 & 102.85 & 3.08 & 13.20 & 1.29 & & 72.38 & & & & 90.69 & 4.89 & 4.94 & & & 541.82 \\
\hline & & V & 132.18 & 58.54 & 1.93 & 6.80 & 0.72 & & 34.33 & & & & 50.57 & 2.99 & 2.86 & & & 290.92 \\
\hline & & $\Delta_{S}$ & 45.87 & 18.98 & 0.57 & 2.44 & 0.24 & & 13.36 & & & & 16.74 & 0.90 & 0.91 & & & 100.00 \\
\hline & III $b$ & $k$ & 159 & 120 & 6 & 12 & 2 & & 24 & & & & 48 & 7 & 2 & & 4 & 384 \\
\hline & & $d_{\min }$ & 2.29 & 2.87 & 3.47 & 2.97 & 3.29 & & 2.04 & & & & 2.89 & 3.50 & 3.32 & & 4.40 & 2.04 \\
\hline & & $d_{\max }$ & 4.99 & 4.74 & 4.55 & 4.53 & 3.55 & & 4.53 & & & & 4.66 & 3.91 & 3.71 & & 4.78 & 4.99 \\
\hline & & $S$ & 260.00 & 96.93 & 3.08 & 9.19 & 1.29 & & 50.47 & & & & 108.35 & 4.89 & 4.94 & & 5.76 & 544.89 \\
\hline & & $V$ & 136.82 & 54.16 & 1.93 & 5.24 & 0.72 & & 23.17 & & & & 60.76 & 2.99 & 2.86 & & 4.27 & 292.92 \\
\hline & & $\Delta_{S}$ & 47.72 & 17.79 & 0.56 & 1.69 & 0.24 & & 9.26 & & & & 19.89 & 0.90 & 0.91 & & 1.06 & 100.00 \\
\hline
\end{tabular}


Table S3 (continued)

\begin{tabular}{|c|c|c|c|c|c|c|c|c|c|c|c|c|c|c|c|c|c|c|}
\hline Form & Refcode & & $\mathrm{H} / \mathrm{H}$ & $\mathrm{H} / \mathrm{C}$ & $\mathrm{C} / \mathrm{C}$ & $\mathrm{H} / \mathrm{N}$ & $\mathrm{C} / \mathrm{N}$ & $\mathrm{N} / \mathrm{N}$ & $\mathrm{H} / \mathrm{O}$ & $\mathrm{C} / \mathrm{O}$ & $\mathrm{N} / \mathrm{O}$ & $\mathrm{O} / \mathrm{O}$ & $\mathrm{H} / \mathrm{Cl}$ & $\mathrm{C} / \mathrm{Cl}$ & $\mathrm{N} / \mathrm{Cl}$ & $\mathrm{O} / \mathrm{Cl}$ & $\mathrm{Cl} / \mathrm{Cl}$ & $\Sigma$ \\
\hline \multirow[t]{18}{*}{ IV } & MELFIT04 & $k$ & 163 & 118 & 13 & 13 & 5 & & 26 & 7 & & & 41 & 12 & 2 & 4 & 1 & 404 \\
\hline & & $d_{\min }$ & 2.21 & 2.87 & 3.58 & 2.85 & 3.61 & & 2.09 & 3.63 & & & 2.91 & 3.62 & 3.95 & 3.58 & 3.55 & 2.09 \\
\hline & & $d_{\text {max }}$ & 4.95 & 4.56 & 4.12 & 3.88 & 4.20 & & 4.26 & 4.22 & & & 4.92 & 4.47 & 3.97 & 4.07 & 3.55 & 4.95 \\
\hline & & $S$ & 259.04 & 87.68 & 5.99 & 18.25 & 1.57 & & 53.86 & 2.78 & & & 89.90 & 10.51 & 0.34 & 4.03 & 2.06 & 536.01 \\
\hline & & $V$ & 133.88 & 49.67 & 3.80 & 9.53 & 0.97 & & 25.47 & 1.74 & & & 49.20 & 6.61 & 0.23 & 2.42 & 1.22 & 284.73 \\
\hline & & $\Delta_{S}$ & 48.33 & 16.36 & 1.12 & 3.40 & 0.29 & & 10.05 & 0.52 & & & 16.77 & 1.96 & 0.06 & 0.75 & 0.38 & 100.00 \\
\hline & $\mathrm{IV} a$ & $k$ & 168 & 105 & 15 & 14 & 6 & & 34 & 9 & & & 32 & 11 & 3 & 5 & & 402 \\
\hline & & $d_{\min }$ & 2.21 & 2.87 & 3.58 & 2.85 & 3.61 & & 2.09 & 3.63 & & & 2.94 & 3.75 & 3.95 & 3.58 & & 2.09 \\
\hline & & $d_{\max }$ & 4.44 & 4.56 & 4.12 & 3.40 & 4.20 & & 4.26 & 4.22 & & & 4.31 & 4.47 & 3.97 & 4.07 & & 4.95 \\
\hline & & $S$ & 260.43 & 86.77 & 6.32 & 19.53 & 1.57 & & 62.98 & 5.25 & & & 72.52 & 7.99 & 0.42 & 6.67 & & 530.44 \\
\hline & & $V$ & 131.55 & 47.78 & 4.02 & 9.80 & 0.97 & & 29.99 & 3.27 & & & 39.47 & 5.08 & 0.28 & 3.99 & & 276.18 \\
\hline & & $\Delta_{S}$ & 49.10 & 16.36 & 1.19 & 3.68 & 0.30 & & 11.87 & 0.99 & & & 13.67 & 1.51 & 0.08 & 1.26 & & 100.00 \\
\hline & $\mathrm{IV} b$ & $k$ & 158 & 131 & 10 & 12 & 4 & & 18 & 5 & & & 50 & 13 & 1 & 3 & 1 & 406 \\
\hline & & $d_{\text {min }}$ & 2.21 & 2.96 & 3.58 & 2.90 & 3.61 & & 2.09 & 3.84 & & & 2.91 & 3.62 & 3.97 & 3.59 & 3.55 & 2.09 \\
\hline & & $d_{\text {max }}$ & 4.95 & 4.56 & 4.12 & 3.88 & 4.01 & & 4.26 & 4.06 & & & 4.92 & 4.47 & 3.97 & 4.07 & 3.55 & 4.95 \\
\hline & & $S$ & 257.65 & 88.58 & 5.65 & 16.98 & 1.57 & & 44.73 & 0.32 & & & 107.27 & 13.04 & 0.26 & 1.40 & 4.12 & 541.57 \\
\hline & & $V$ & 136.22 & 51.56 & 3.58 & 9.27 & 0.97 & & 20.94 & 0.21 & & & 58.94 & 8.15 & 0.17 & 0.84 & 2.44 & 293.28 \\
\hline & & $\Delta_{S}$ & 47.57 & 16.36 & 1.04 & 3.13 & 0.29 & & 8.26 & 0.06 & & & 19.81 & 2.41 & 0.05 & 0.26 & 0.76 & 100.00 \\
\hline \multirow[t]{6}{*}{$\mathrm{V}$} & MELFIT05 & $k$ & 166 & 112 & 10 & 14 & 4 & & 24 & 4 & & & 40 & 14 & 2 & 2 & & 392 \\
\hline & & $d_{\min }$ & 2.57 & 2.80 & 3.43 & 2.76 & 3.85 & & 2.06 & 3.77 & & & 2.87 & 3.64 & 3.42 & 3.66 & & 2.06 \\
\hline & & $d_{\max }$ & 4.64 & 4.66 & 4.95 & 3.92 & 4.10 & & 3.84 & 3.92 & & & 4.46 & 5.01 & 3.42 & 3.66 & & 5.01 \\
\hline & & $S$ & 258.70 & 102.08 & 2.23 & 14.15 & 0.90 & & 55.51 & 1.80 & & & 92.78 & 7.92 & 2.88 & 2.51 & & 541.46 \\
\hline & & V & 137.66 & 55.14 & 1.51 & 7.32 & 0.59 & & 25.33 & 1.13 & & & 50.81 & 5.56 & 1.64 & 1.53 & & 288.23 \\
\hline & & $\Delta_{S}$ & 47.78 & 18.85 & 0.41 & 2.61 & 0.17 & & 10.25 & 0.33 & & & 17.14 & 1.46 & 0.53 & 0.46 & & 100.00 \\
\hline
\end{tabular}


Table S3 (continued)

\begin{tabular}{|c|c|c|c|c|c|c|c|c|c|c|c|c|c|c|c|c|c|c|}
\hline Form & Refcode & & $\mathrm{H} / \mathrm{H}$ & $\mathrm{H} / \mathrm{C}$ & $\mathrm{C} / \mathrm{C}$ & $\mathrm{H} / \mathrm{N}$ & $\mathrm{C} / \mathrm{N}$ & $\mathrm{N} / \mathrm{N}$ & $\mathrm{H} / \mathrm{O}$ & $\mathrm{C} / \mathrm{O}$ & $\mathrm{N} / \mathrm{O}$ & $\mathrm{O} / \mathrm{O}$ & $\mathrm{H} / \mathrm{Cl}$ & $\mathrm{C} / \mathrm{Cl}$ & $\mathrm{N} / \mathrm{Cl}$ & $\mathrm{O} / \mathrm{Cl}$ & $\mathrm{Cl} / \mathrm{Cl}$ & $\Sigma$ \\
\hline \multirow[t]{18}{*}{ VI } & MELFIT06 & $k$ & 149 & 122 & 15 & 20 & 1 & & 29 & 5 & 1 & 1 & 37 & 20 & & 1 & 2 & 404 \\
\hline & & $d_{\min }$ & 2.15 & 2.71 & 3.75 & 2.76 & 3.52 & 3.75 & 1.95 & 3.76 & 3.83 & 4.23 & 2.80 & 3.30 & & 4.16 & 3.74 & 1.95 \\
\hline & & $d_{\max }$ & 4.78 & 4.64 & 4.58 & 3.92 & 3.52 & 3.75 & 4.41 & 4.28 & 3.83 & 4.23 & 4.70 & 4.90 & & 4.16 & 3.92 & 4.90 \\
\hline & & $S$ & 255.20 & 89.35 & 6.85 & 19.28 & 0.80 & 0.04 & 59.62 & 2.93 & 0.00 & 0.19 & 87.77 & 8.99 & & 1.25 & 4.29 & 536.56 \\
\hline & & $V$ & 131.09 & 50.40 & 4.74 & 9.76 & 0.47 & 0.02 & 29.19 & 1.91 & 0.00 & 0.13 & 48.51 & 6.00 & & 0.87 & 2.75 & 285.83 \\
\hline & & $\Delta_{S}$ & 47.56 & 16.65 & 1.28 & 3.59 & 0.15 & 0.01 & 11.11 & 0.55 & $<0.001$ & 0.04 & 16.36 & 1.68 & & 0.23 & 0.80 & 100.00 \\
\hline & $\mathrm{VI} a$ & $k$ & 148 & 123 & 20 & 21 & 1 & 1 & 33 & 7 & 2 & 1 & 35 & 13 & & 1 & 2 & 408 \\
\hline & & $d_{\min }$ & 2.50 & 2.71 & 3.75 & 2.76 & 3.52 & 3.75 & 2.00 & 3.76 & 3.83 & 4.23 & 2.80 & 3.77 & & 4.16 & 3.74 & 2.00 \\
\hline & & $d_{\max }$ & 4.78 & 4.31 & 4.40 & 3.92 & 3.52 & 3.75 & 4.41 & 4.28 & 3.83 & 4.23 & 4.70 & 4.57 & & 4.16 & 3.92 & 4.78 \\
\hline & & $S$ & 254.39 & 84.42 & 9.85 & 20.20 & 0.80 & 0.03 & 66.89 & 4.46 & 0.00 & 0.19 & 81.32 & 5.93 & & 1.25 & 4.29 & 534.02 \\
\hline & & V & 130.52 & 47.66 & 6.73 & 10.04 & 0.47 & 0.02 & 33.09 & 2.85 & 0.00 & 0.13 & 45.20 & 3.97 & & 0.87 & 2.75 & 284.30 \\
\hline & & $\Delta_{S}$ & 47.64 & 15.81 & 1.84 & 3.78 & 0.15 & 0.01 & 12.53 & 0.84 & 0.00 & 0.04 & 15.23 & 1.11 & & 0.23 & 0.80 & 100.00 \\
\hline & VI $b$ & $k$ & 150 & 121 & 10 & 19 & 1 & 1 & 25 & 3 & & 1 & 39 & 27 & & 1 & 2 & 400 \\
\hline & & $d_{\min }$ & 2.15 & 2.94 & 3.75 & 2.82 & 3.52 & 3.75 & 1.95 & 4.25 & & 4.23 & 2.80 & 3.30 & & 4.16 & 3.74 & 1.95 \\
\hline & & $d_{\text {max }}$ & 4.78 & 4.64 & 4.58 & 3.92 & 3.52 & 3.75 & 4.41 & 4.25 & & 4.23 & 4.38 & 4.90 & & 4.16 & 3.92 & 4.90 \\
\hline & & $S$ & 256.02 & 94.29 & 3.84 & 18.35 & 0.80 & 0.03 & 52.36 & 1.40 & & 0.19 & 94.23 & 12.05 & & 1.25 & 4.29 & 539.10 \\
\hline & & $V$ & 131.66 & 53.14 & 2.74 & 9.47 & 0.47 & 0.02 & 25.28 & 0.99 & & 0.13 & 51.83 & 8.03 & & 0.87 & 2.75 & 287.38 \\
\hline & & $\Delta_{S}$ & 47.49 & 17.49 & 0.71 & 3.40 & 0.15 & 0.01 & 9.71 & 0.26 & & 0.04 & 17.48 & 2.24 & & 0.23 & 0.80 & 100.00 \\
\hline \multirow[t]{6}{*}{ VII } & MELFIT07 & $k$ & 154 & 98 & 22 & 10 & 12 & & 36 & 6 & & 1 & 36 & 14 & & & 3 & 392 \\
\hline & & $d_{\min }$ & 2.33 & 2.70 & 3.59 & 3.13 & 3.53 & & 2.01 & 3.48 & & 3.37 & 3.03 & 3.73 & & & 3.50 & 2.01 \\
\hline & & $d_{\max }$ & 4.73 & 3.99 & 4.23 & 3.80 & 4.05 & & 3.92 & 3.77 & & 3.37 & 4.14 & 4.10 & & & 3.53 & 4.73 \\
\hline & & $S$ & 261.27 & 72.91 & 13.29 & 9.62 & 3.02 & & 57.49 & 2.39 & & 0.71 & 72.84 & 11.03 & & & 12.05 & 516.62 \\
\hline & & $V$ & 129.86 & 37.83 & 8.28 & 5.31 & 1.88 & & 27.79 & 1.42 & & 0.40 & 40.69 & 7.13 & & & 7.05 & 267.62 \\
\hline & & $\Delta_{S}$ & 50.57 & 14.11 & 2.57 & 1.86 & 0.58 & & 11.13 & 0.46 & & 0.14 & 14.10 & 2.14 & & & 2.33 & 100.00 \\
\hline \multirow[t]{6}{*}{ VIII } & MELFIT18 & $k$ & 144 & 122 & 12 & 10 & 6 & & 26 & 12 & & & 46 & 2 & 4 & 6 & & 390 \\
\hline & & $d_{\min }$ & 2.49 & 2.85 & 3.30 & 3.03 & 3.55 & & 1.94 & 3.57 & & & 2.85 & 3.38 & 3.64 & 3.62 & & 2.01 \\
\hline & & $d_{\max }$ & 4.37 & 4.25 & 3.77 & 3.83 & 3.89 & & 4.50 & 4.06 & & & 4.67 & 3.38 & 4.02 & 3.92 & & 4.73 \\
\hline & & $S$ & 257.51 & 90.47 & 4.08 & 11.65 & 3.80 & & 46.61 & 6.67 & & & 91.09 & 3.59 & 2.06 & 4.79 & & 522.32 \\
\hline & & $V$ & 127.91 & 48.45 & 2.43 & 6.00 & 2.27 & & 21.06 & 4.07 & & & 47.40 & 2.03 & 1.25 & 2.97 & & 265.82 \\
\hline & & $\Delta_{S}$ & 49.30 & 17.32 & 0.78 & 2.23 & 0.73 & & 8.92 & 1.28 & & & 17.44 & 0.69 & 0.39 & 0.92 & & 100.00 \\
\hline \multirow[t]{6}{*}{ IX } & MELFIT19 & $k$ & 144 & 130 & 2 & 16 & & & 22 & 6 & 2 & 2 & 46 & 12 & 2 & 2 & & 386 \\
\hline & & $d_{\text {min }}$ & 2.32 & 2.66 & 3.75 & 3.07 & & & 2.03 & 3.59 & 3.79 & 3.61 & 2.84 & 3.50 & 4.14 & 3.19 & & 2.03 \\
\hline & & $d_{\text {max }}$ & 4.36 & 4.68 & 3.75 & 3.78 & & & 3.87 & 3.69 & 3.79 & 3.61 & 4.20 & 4.31 & 4.14 & 3.19 & & 4.68 \\
\hline & & $S$ & 244.51 & 101.79 & 0.81 & 18.21 & & & 46.63 & 7.05 & 0.12 & 1.17 & 92.42 & 8.09 & 0.55 & 8.62 & & 529.95 \\
\hline & & $V$ & 120.14 & 54.99 & 0.50 & 9.65 & & & 22.10 & 4.27 & 0.07 & 0.70 & 51.51 & 5.30 & 0.38 & 4.58 & & 274.20 \\
\hline & & $\Delta_{S}$ & 46.14 & 19.21 & 0.15 & 3.44 & & & 8.80 & 1.33 & 0.02 & 0.22 & 17.44 & 1.53 & 0.10 & 1.63 & & 100.00 \\
\hline
\end{tabular}

